\title{
Head-pelvis coupling is increased during turning in patients with Parkinson's disease and FOG.
}

Spildooren Joke, Msc; ${ }^{1}$ Vercruysse Sarah, Msc; ${ }^{1}$ Heremans Elke, $\mathrm{PhD} ;{ }^{1}$ Galna Brook, $\mathrm{PhD}^{2}$; Vandenbossche Jochen, Msc; ${ }^{3} \mathrm{PhD}$; Desloovere Kaat, $\mathrm{PhD} ;{ }^{4}$ Vandenberghe Wim, MD, $\mathrm{PhD} ;{ }^{5}$ Nieuwboer Alice, $\mathrm{PhD}^{1}$.

${ }^{1}$ Department of Rehabilitation Sciences, Katholieke Universiteit, Leuven, Belgium

${ }^{2}$ Institute of Ageing and health, Newcastle University, United Kingdom

${ }^{3}$ Neurological Rehabilitation, Vrije universiteit Brussel, Belgium

${ }^{4}$ Clinical Motion Analysis Laboratory, University Hospital Leuven, Belgium

${ }^{5}$ Department of Neurosciences, Katholieke Universiteit Leuven, Belgium

Spildooren Joke

Tervuursevest 101, bus 1501,

3001 Heverlee, Belgium

Tel +3216329030

fax +3216329197

joke.spildooren@faber.kuleuven.be

Word count: 3203

Running title: head-pelvis dissociation during turning in freezers

Keywords: Freezing Of Gait, turning, Parkinson, trunk rotation, en-bloc

Conflict of interest: None

Funding sources for study: Flanders Fund for Scientific Research (project G0691.08) and the Research Council of Katholieke Universiteit Leuven, Belgium (contract OT/07/074) 


\section{Abstract}

Background: Turning is the most important trigger for Freezing Of Gait (FOG) and is characterized by a decreased head-pelvis rotation in the early stages of Parkinson's Disease. Objective: To investigate the influence of impaired head-pelvis rotation during turning on FOG. Methods: Head, trunk and pelvic rotation were measured at the onset and throughout a $180^{\circ}$ turn in 13 Freezers and 14 non-freezers (OFF medication). Fourteen controls turned at preferred and slow speed to investigate the influence of turn velocity on axial rotations. The location and duration of FOG-episodes were defined during the turn. Results: At turning onset, head rotation preceded thorax and pelvic rotation in all groups but this cranio-caudal sequence disappeared when FOG occurred during the trial. The maximum head-pelvis separation was significantly greater in controls compared to freezers and non-freezers (35.4 vs 25.7 and $27.3^{\circ}, \mathrm{p}<0.01$ ), but this finding was speed-dependent. The timing of maximum headpelvis separation was delayed in freezers compared to non-freezers and controls, irrespective of turn velocity. This delay was correlated with increased neck-rigidity $(\mathrm{R}=0.62, \mathrm{p}=0.02)$ and worsened during FOG-trials. FOG occurred more often at the end of the turn, when the difference in rotation velocity between head and pelvis was the greatest. Conclusions: Even after controlling for speed and disease-severity, turning in freezers was characterized by delayed head rotation and a closer coupling between head and pelvis especially in turns where FOG occurred. These changes may be attributed to delayed preparation for the change in walking direction and as such contribute to FOG. 


\section{Introduction}

Freezing Of Gait (FOG) is defined as "a brief, episodic absence or marked reduction of forward progression of the feet despite the intention to walk" ${ }^{\prime 1}$ and described by patients as if their feet are glued to the floor ${ }^{2}$. FOG is not associated with tremor ${ }^{3}$ but is regarded as a central symptom in the postural instability and gait difficulty (PIGD) phenotype of $\mathrm{PD}^{4,5}$. The underlying mechanisms are still poorly understood ${ }^{1}$.

FOG occurs especially during turning ${ }^{6}$ and $94 \%$ of patients who freeze frequently report turning difficulties ${ }^{7}$. The turn is initiated in a cranio-caudal sequence (i.e. the head rotates first, then the trunk and finally the feet $)^{8}$ inducing axial rotation. Straight-line gait in PD is characterized by more "en-bloc" movement ${ }^{9,10}$, which is partially related to the reduced gait velocity $^{11,12,13}$ and might also be related to rigidity ${ }^{14}$.

The axial kinematics of head, trunk and pelvis during turning are more demanding compared to linear walking. Patients with early PD and relatively unimpaired straight-line walking turn more slowly, take more steps and have a decreased head-pelvis rotation during turning compared to healthy controls ${ }^{15}$, especially during sharp turns ${ }^{16,17}$. Rapid and full $\left(360^{\circ}\right)$ turns are also characterized by more FOG-episodes ${ }^{18,19}$. Just like $\mathrm{FOG}^{2}$, turning difficulties ${ }^{20}$ and "en-bloc" movement ${ }^{9}$ augment with increasing disease-severity.

If deterioration of axial movement is a contributing factor to freezing, freezers are expected to turn more "en-bloc" compared to non-freezers and controls, especially prior to FOG-episodes and FOG will occur more frequently at the most pivotal point of $180^{\circ}$ turning-arc, i.e. at $90^{\circ}$ degrees when the head-pelvis separation and lateral translation is the greatest and consequently turning becomes more unstable ${ }^{21}$.

This study therefore tackled the following research questions: 1) Is axial movement impairment related to the occurrence of FOG? This question was answered on several levels: the axial rotation was compared between freezers, non-freezers and controls and between 
trials with and without FOG. Further, we determined where in the turning-arc FOG-episodes predominantly occurred. 2) Is axial movement impairment visible during turn preparation and can FOG be predicted at the start of the turn? The onset of head, trunk and pelvis rotation and the spatiotemporal characteristics of the pre-turning steps were compared between groups and between trials with and without FOG. In addition, we explored the speed-dependency and the relation with neck-rigidity of axial movement impairment, as it was shown that these factors determine "en-bloc" movements.

\section{Methods}

\section{Participants}

After approval from the local ethics committee, twenty-seven PD patients were recruited in the Movement Disorders Clinic of the University Hospital Leuven. Inclusion criteria were: Hoehn \& Yahr II-III ${ }^{22}$, no dementia $(\text { MMSE }>24)^{23}$, no comorbidity limiting gait and no STNDBS. The participants were divided into 13 freezers and 14 non-freezers based on the first item of the revised freezing of gait questionnaire (NFOG-Q) ${ }^{24}$. Groups were matched for age and disease-severity (H\&Y and UPDRS III $^{25}$ ). Fourteen age-matched controls were also recruited.

\section{Experimental protocol}

Participants were tested after overnight medication withdrawal (OFF-period). The protocol included a 5 meter walk towards two retroreflective markers placed 0.5 meters away from each other as previously described ${ }^{18}$ (Figure 1). All participants were asked to turn $180^{\circ}$ towards both sides at random (around the turning markers). Turning towards the left and the right side was pooled. The small and unobtrusive markers were positioned so that the turningarc was standardized without suggesting a space limitation (which could elicit $\mathrm{FOG}^{6}$ ). Turning was performed with and without an auditory cognitive dual-task (DT) (color 
classification $\operatorname{task}^{18,26,27}$ ) in a counterbalanced order. The participants were instructed to answer 'yes' to a criterion color and 'no' to another color, which were presented verbally by a prerecorded audiotape in a random order every 2 seconds ${ }^{18}$.

Further, to investigate the possible effect of turn velocity on group differences, eleven controls were tested at preferred speed as well as at a speed matched to the freezers. These controls were asked to match the heel contact with a cue at a frequency of $74 \mathrm{steps} / \mathrm{min}$. This imposed cadence was calculated as $0.76 \times$ required speed according to Osaki et $\mathrm{al}^{28}$. Every condition was performed 3 times.

\section{Equipment}

An eight camera VICON data capturing system (Vicon Motion Systems, Workstation 612) was positioned around a $10 \mathrm{~m}$ walkway. Thirty-one retroreflective markers $(14 \mathrm{~mm}$ in diameter) were placed on the head, thorax, pelvis and upper and lower extremities according to the full body plug-in-gait model (Oxford Metrics, Oxford, UK, sampling frequency $=50 \mathrm{~Hz}$ ), allowing us to investigate the position of the Center Of Mass (COM) and the rotation of the body segments during turning.

\section{Outcome measures}

The pelvis, thorax and head segments were defined according to the Vicon plug-in-gait model. The orientation of each segment relative to the vertical axis of the laboratory coordinate system was extracted to define turning, whereby a segment had an orientation of approximately $0^{\circ}$ when walking towards the retroreflective marker and $180^{\circ}$ after turning back.

The following parameters were calculated: 
1) FOG-occurrence: The start of every FOG-episode was defined by the onset of a hesitation or interruption of movement (as previously described $)^{29}$ and located in the turn;

2) Onset of head, trunk and pelvic rotation: The distance from the position of COM to the retroflective turning markers placed on the floor at the time that head, trunk or pelvis reached $10^{\circ}$ rotation (expressed in $\mathrm{mm}$ ). The threshold of $10^{\circ}$ was chosen to ensure that rotation was a result of turn-initiation instead of the natural fluctuations of head pelvis during straight-line walking ${ }^{30}$. A negative value means that the rotation started before the COM reached the turning marker (Figure 1);

3) Head-pelvis separation: angular difference $\left(^{\circ}\right)$ between head and pelvis, calculated for every $5^{\circ}$ of pelvic rotation (from 10 to $170^{\circ}$ ). This resulted in 33 data points equally separated from each other in all participants.

4) Normalized head-pelvis velocity difference: The difference in angular velocity of head and pelvis rotation (expressed as a percentage of pelvic rotation), calculated for every $5^{\circ}$ of pelvic rotation. A positive value equals a higher rotation velocity of the head compared to the pelvis. A negative value refers to the counter-rotation (i.e. head and pelvis rotating in the opposite direction). This counter-rotation is initiated at the second part of the turn, to prepare for normal gait (Figure 1). 


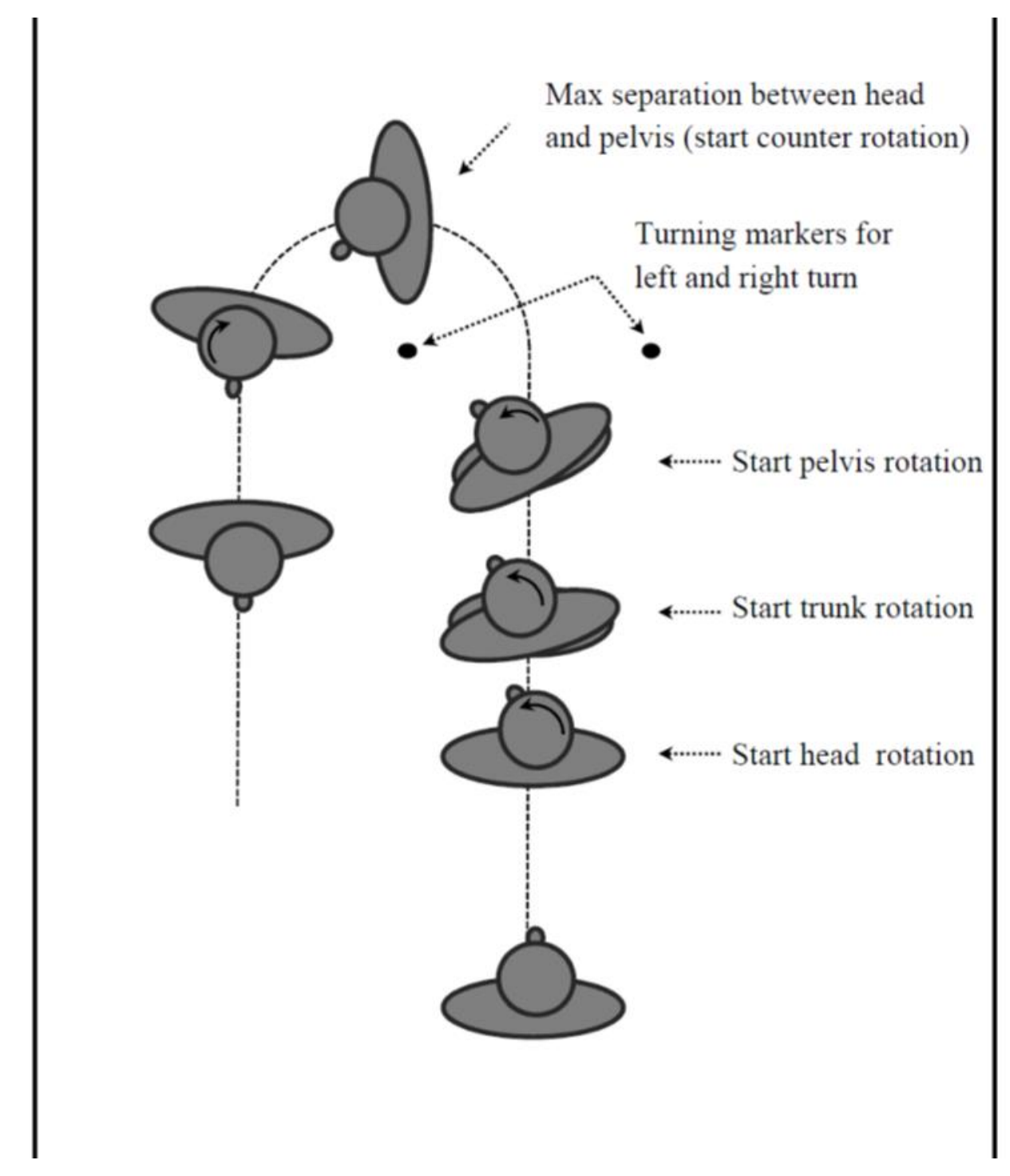

Figure 1: Top view of the walkway. Subjects were asked to turn around the left or right turning marker. A normal turn was initiated with rotation of the head, followed by trunk and pelvis. The turn onset of each segment was defined as the distance from the position of COM to the turning marker at the start of the rotation. The turning arrays describe the turn direction of the head in relation to the pelvis. During mid-turn the maximum head-pelvis separation is reached and the counter-rotation of the head is initiated.

5) Average turn velocity: calculated as $160^{\circ}$ pelvic rotation divided by the total time needed to rotate the pelvis from 10 to $170^{\circ}$ (expressed as $\%$ s);

6) Turning depth: distance from COM to the turning markers placed on the floor at the apex of the turn (expressed in $\mathrm{mm}$ ); 


\section{Data-analysis}

Clinical characteristics were analyzed with a one-way ANOVA (age and leg length), KruskalWallis (MMSE), t-test (UPDRS III) and Mann-Whitney U tests (disease-duration, H\&Y and neck-rigidity item of UPDRS).

Kinematic data were calculated as an average over six trials per condition (left and right turns pooled). When FOG occurred during the protocol, the trial was excluded from group analyses and an average was calculated for the remaining trials. A mixed model ANOVA (group*DT) was used to check whether the dual-tasking influenced axial kinematics. Post-hoc NewmanKeuls tests were executed when significant differences were found. Dissociation curves of all trials (head-pelvis separation and normalized velocity) were analyzed graphically and compared statistically per $5^{\circ}$ pelvic rotation using Bonferroni correction for the 33 datapoints.

Trials with and without FOG were analyzed within the group of freezers who actually froze during the protocol $(n=5)$ using the non-parametric Wilcoxon signed rank test. All statistical analyses were performed using Statistica (version 9.0) and levels of significance were set at $\alpha=0.05$.

\section{Results}

\section{Descriptive results}

Table 1: Subject characteristics: mean (SD) measured during ON-phase of the medication cycle.

\begin{tabular}{lllll}
\hline & $\begin{array}{l}\text { Controls } \\
(\mathbf{n = 1 4 )}\end{array}$ & $\begin{array}{l}\text { Non-freezers } \\
(\mathbf{n = 1 4 )}\end{array}$ & $\begin{array}{l}\text { Freezers } \\
(\mathbf{n}=13)\end{array}$ & p-value \\
\hline Age (years) & $65.2(6.8)$ & $66.7(7.4)$ & $68.1(7.5)$ & .59 \\
Leg length (cm) & $90.1(4.9)$ & $88.9(6.5)$ & $88.5(4.5)$ & .73 \\
MMSE & $29.1(1.3)^{*}$ & $28.7(1.2)$ & $27.7(1.2)$ & .01 \\
Disease duration (years) & & $7.8(4.8)$ & $9.0(5.0)$ & .30 \\
H\&Y & & $2.4(0.3)$ & $2.5(0.5)$ & .42 \\
UPDRS III & & $34.4(9.9)$ & $38.7(14.2)$ & .37 \\
Neck rigidity & $2.1(1.8)$ & $2.8(1.1)$ & .31 \\
Fall-frequency & & $1 / 14$ & $5 / 13$ & \\
\hline
\end{tabular}

Fall frequency is the number of patients that had been fallen in the past six months.

*Freezers and controls were significantly different at $\mathrm{p}<0.05$ (Kruskal-Wallis). 
Clinical characteristics were comparable for the different groups, except for MMSE, which was significantly worse in freezers than controls, but not compared to non-freezers. Further, five freezers had fallen in the past six months compared to only one non-freezer (Table 1).

The turning depth was similar in the 3 groups $(\mathrm{p}=0.48)$. Therefore, a good standardization of the turning-arc was accomplished (Figure 2).

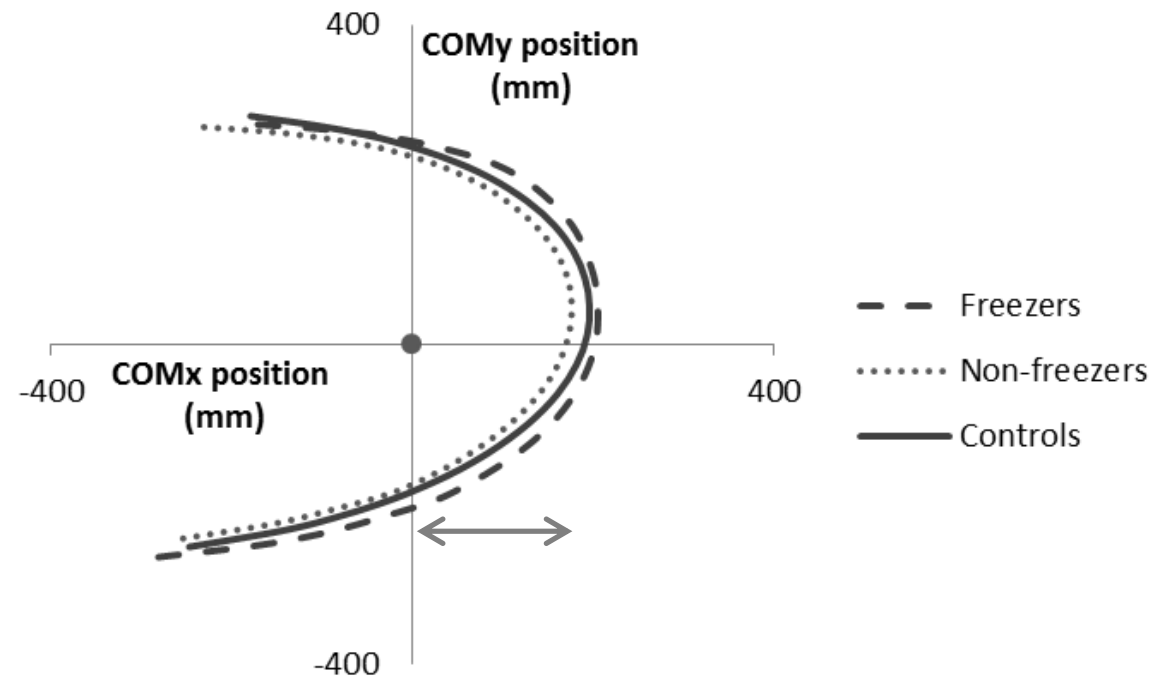

Figure 2: Turning-arc: Distance from COM to the turning marker placed on the ground $(\bullet)$ for freezers, nonfreezers and controls. The turning depth is marked by the grey line. No significant differences for turning depth were found.

No interaction effect of group*DT was found for any of the head, trunk and pelvis kinematic data. Trials with and without DT were therefore pooled for further analysis.

Twenty-nine FOG episodes (14 episodes during DT and 15 during noDT) were detected, in 5 freezers $(10,8,8,2$ and 1 FOG-episodes for the different freezers). FOG occurred especially at the end of the turn (11 FOG-episodes occurred after $160^{\circ}$ pelvic rotation) (see Figure $3 \mathrm{~A}$ ). These episodes were characterized by a shorter FOG-duration $(0.63 \mathrm{sec}$ vs. $3.71 \mathrm{sec}, \mathrm{p}=0.003)$ compared to freezing-episodes earlier in the turn. 


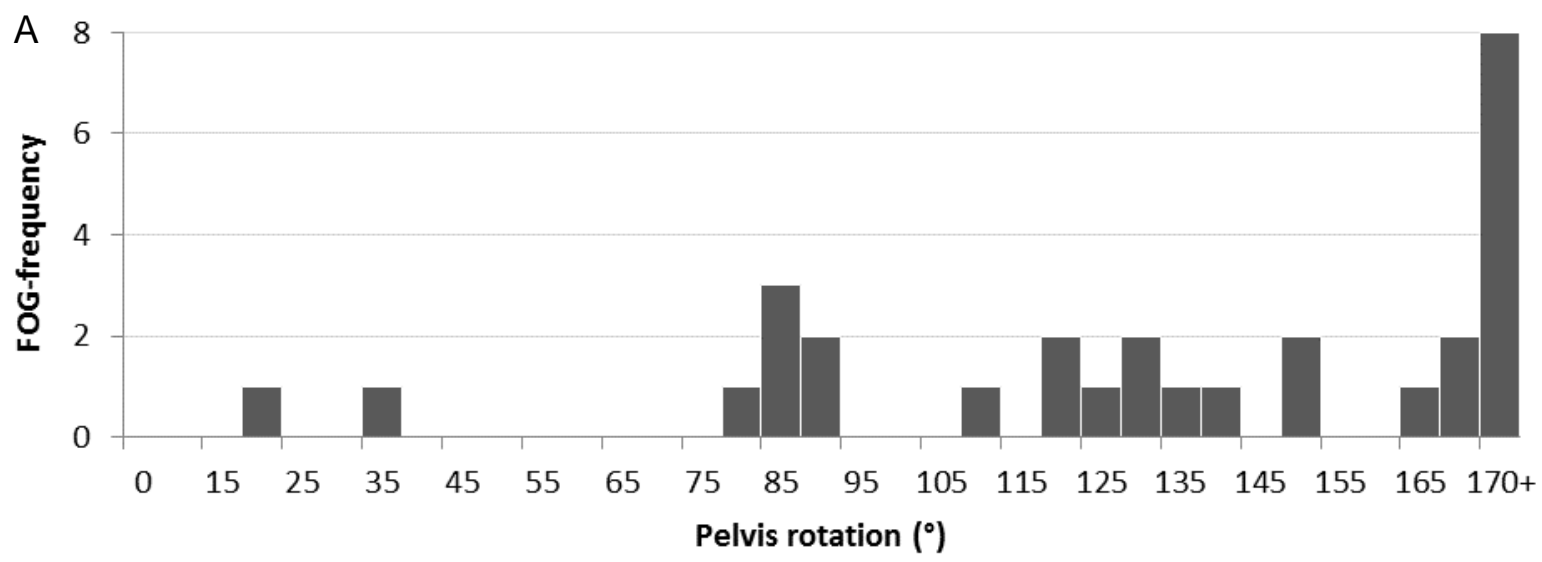

B

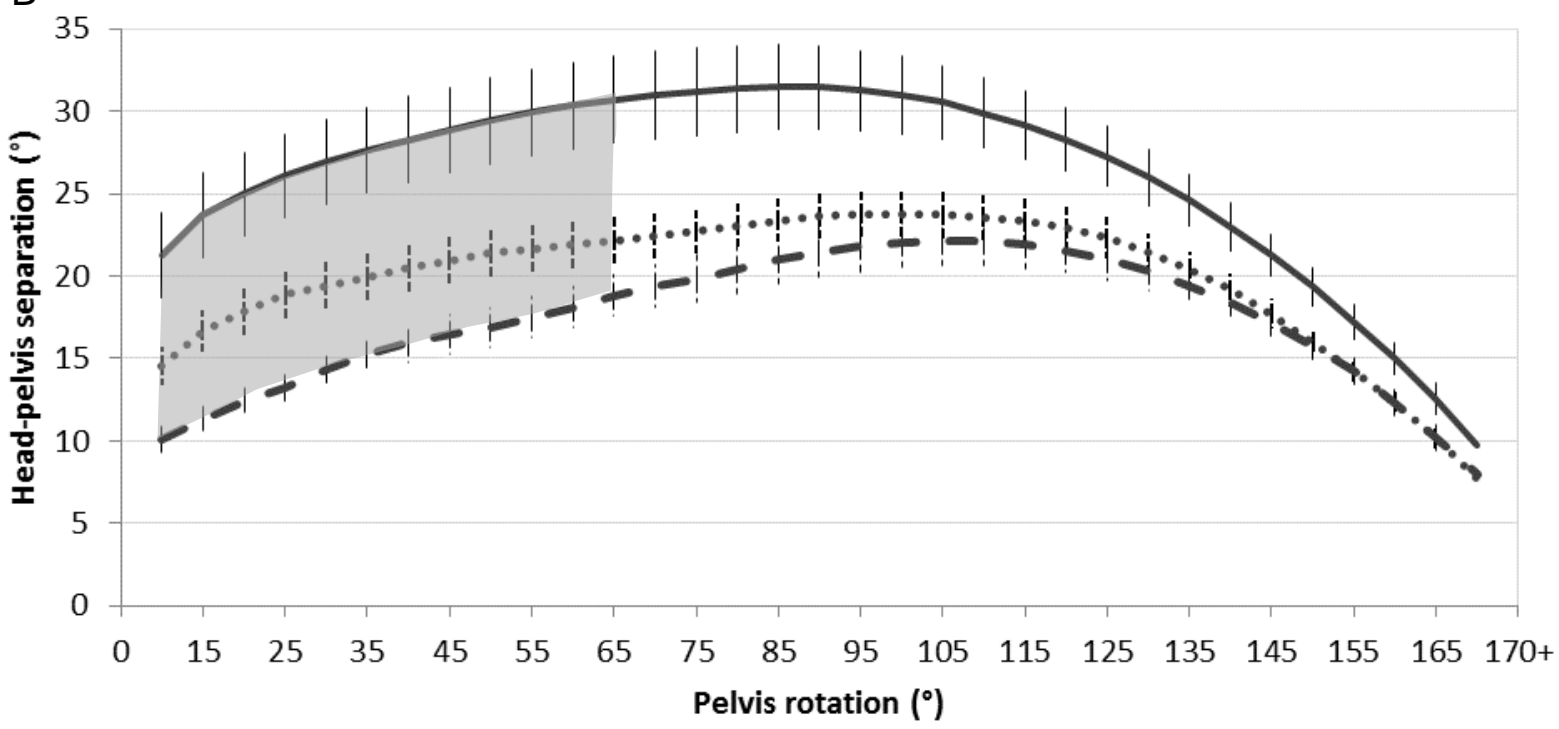

C

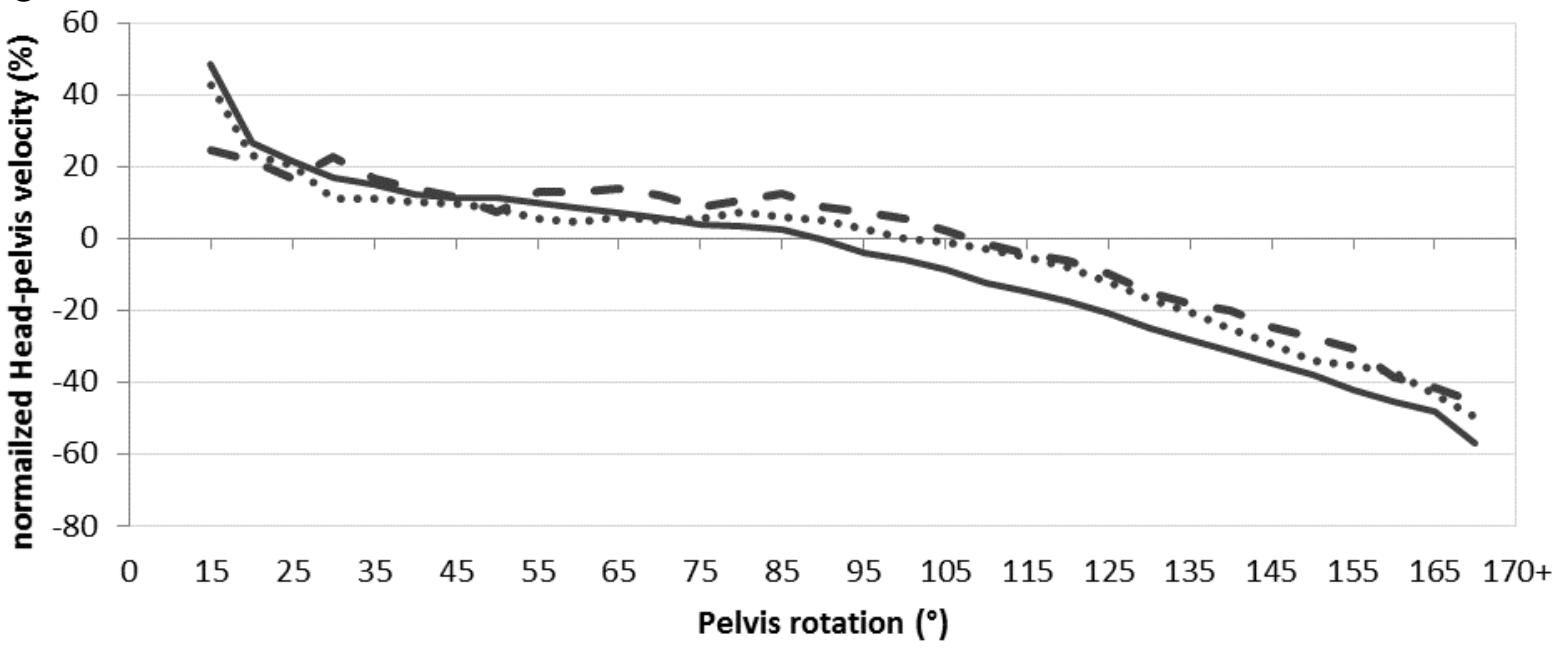

Figure 3: FOG-frequency for every $5^{\circ}$ of pelvic rotation (A). Figure $\mathrm{B}$ and $\mathrm{C}$ visualizes the Head-pelvis separation (B) and normalized velocity (C) during turning between Freezers $\mathbf{c}-\mathbf{~}$, non-freezers $\cdots \cdots \cdot$ and controls $\longrightarrow$. The grey area visualizes the part of the turn that is significantly different $(p<0.05)$ for freezers compared to controls. 


\section{Turn preparation}

A significant interaction ( $\mathrm{p}<0.01$ ) of group*body part (head, trunk and pelvis) showed that the distance of COM to the turning marker at the start of head rotation was significantly larger in controls $(819.8 \mathrm{~mm})$ compared to non-freezers $(656.6 \mathrm{~mm}, \mathrm{p}=0.01)$, whom in turn started to rotate their head earlier than freezers $(520.3 \mathrm{~mm}, \mathrm{p}=0.04)$ (Figure $4 \mathrm{~A})$. Controls displayed a higher turn velocity than non-freezers and freezers (ANOVA: $95.4 \% \mathrm{sec}$ vs $74.1 \% \mathrm{sec}$ vs $\left.60.6^{\circ} / \mathrm{sec}, \mathrm{p}<0.01\right)$. When controls turned at the same speed as freezers, data confirmed that the onset of the head $(792.7$ vs $350.2 \mathrm{~mm}$ in front of the turning marker, $\mathrm{p}<0.01)$ and pelvis rotation $(256.8$ vs $206.0 \mathrm{~mm}$ in front of the turning marker, $\mathrm{p}<0.01)$ was speed-dependent and thus considerably delayed in controls during the slow condition (Figure 4B). Furthermore, no significant differences were found between in the initiation of head and pelvis rotation between freezers and controls when walking at comparable velocities.

Head rotation preceded thorax and pelvic rotation in all groups $(\mathrm{p}<0.01)$ and in both velocity conditions in controls. However, a within-subgroup analysis in the freezers who froze showed that this cranio-caudal sequence disappeared when FOG occurred during the turn, as head rotation did not precede pelvic rotation in FOG-trials $\left(\mathrm{X}^{2}=1.2, \mathrm{p}=0.55\right)$ whereas it did in trials without FOG $\left(\mathrm{X}^{2}=8.4, \mathrm{p}=0.01\right)$ (Figure $\left.4 \mathrm{C}\right)$.

To check whether pre-freezing characteristics were already apparent before turning initiation, we conducted a supplementary analysis on the two steps preceding the turn (onset turn at $10^{\circ}$ pelvic rotation). No differences in cadence, step length, step time and step width of these two steps were found between trials with and without FOG (Table 2). Pre-turning velocity tended to be slower during FOG-trials (0.68 vs $0.73 \mathrm{~m} / \mathrm{sec}, \mathrm{p}=0.08)$. 
Table 2: Mean (SD) of the Pre-turn steps in trials with and without FOG.

\begin{tabular}{llll}
\hline & No FOG & FOG & p-value \\
\hline Cadence (steps/min) & $61.2(5.0)$ & $59.7(3.9)$ & .69 \\
Step length (m) & $0.36(0.03)$ & $0.34(0.04)$ & .22 \\
Step time $(\mathrm{sec})$ & $0.51(0.04)$ & $0.52(0.03)$ & .89 \\
Step width $(\mathrm{m})$ & $0.14(0.02)$ & $0.14(0.01)$ & .50 \\
Velocity $(\mathrm{m} / \mathrm{sec})$ & $0.73(0.05)$ & $0.68(0.06)$ & .08 \\
\hline
\end{tabular}

Sub-analysis within the 5 freezers who froze during the protocol (Wilcoxon).

\section{Axial movement during turning}

The maximum head-pelvis separation was significantly greater in controls compared to freezers and non-freezers (35.4 vs 25.7 and $\left.27.3^{\circ}, \mathrm{p}<0.01\right)$. This finding was speed-dependent as the maximum separation in controls decreased significantly when they turned slowly $\left(34.8^{\circ}\right.$ vs $\left.24.9^{\circ}, \mathrm{p}<0.01\right)$ and consequently matched the values of freezers $\left(24.9^{\circ}\right.$ vs $\left.25.7^{\circ}, \mathrm{p}=0.83\right)$.

Controls and non-freezers reached maximum head-pelvis separation significantly earlier in the turn than freezers (at $69.3^{\circ}$ and $79.4^{\circ}$ vs $93.2^{\circ}$ of pelvic rotation, $\mathrm{p}<0.01$ and $\mathrm{p}=0.05$ ) irrespective of turn velocity (timing of maximum separation in controls at $69.8^{\circ}$ during slow and at $71.2^{\circ}$ pelvic rotation during normal speed, $\mathrm{p}=0.86$ ).

Head-pelvis separation in controls was significantly greater than in freezers from the beginning of the turn till $65^{\circ}$ pelvic rotation was reached (Figure 3B).

No group differences in angular head-pelvis velocity were found during the turn. In all groups, the head rotated on average $38.9 \%$ faster than the pelvis in the beginning of the turn (to reach maximum separation), but head velocity decreased $(\mathrm{R}=-.94, \mathrm{p}<0.01)$ towards the end of the turn. The head rotated $50.8 \%$ slower than the pelvis (i.e. counter-rotation) at $170^{\circ}$ pelvic rotation. Therefore, the difference in angular velocity between head and pelvis was the highest at the end of the turn (Figure 3C).

Within-subject analysis of the 5 freezers who froze showed no differences in the maximum head-pelvis dissociation between trials with and without FOG (23.3 vs $\left.21.5^{\circ}, \mathrm{p}=0.89\right)$. The 
A

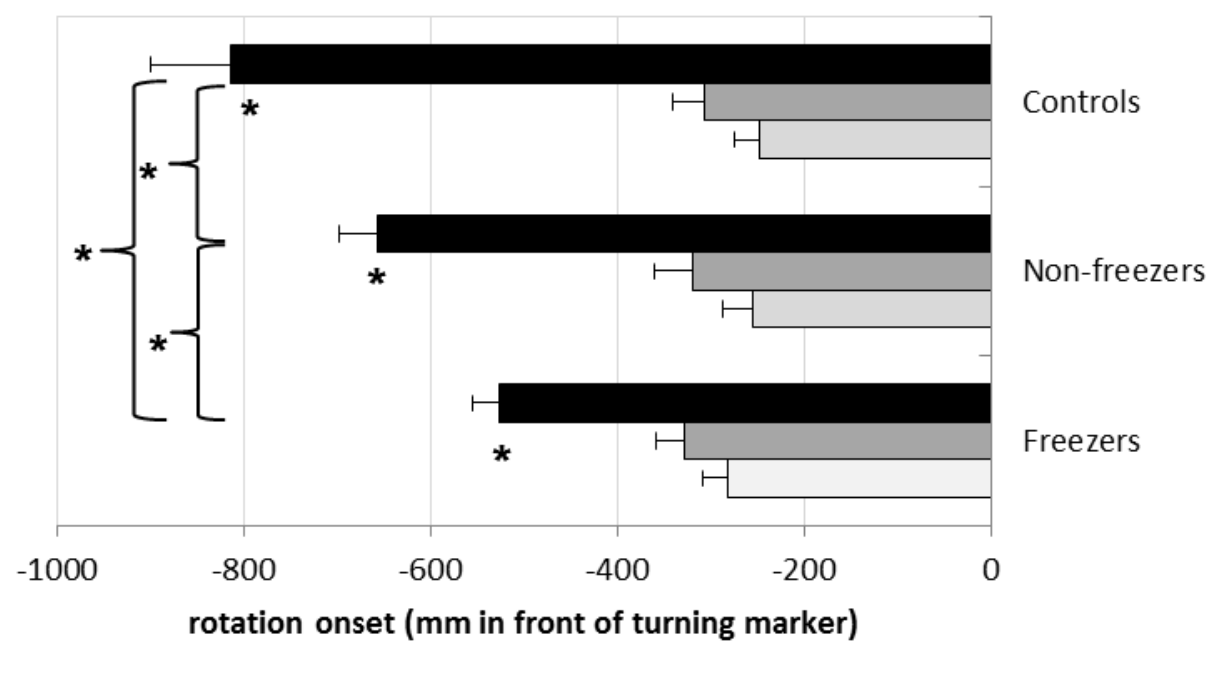

B

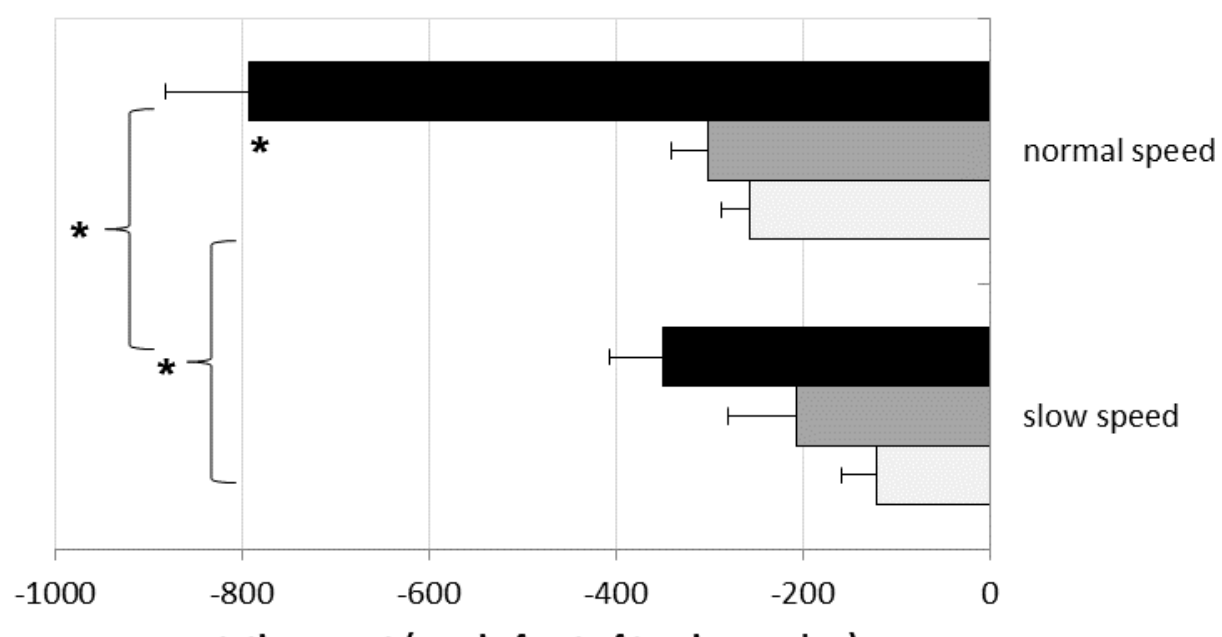

rotation onset ( $\mathrm{mm}$ in front of turning marker)

C

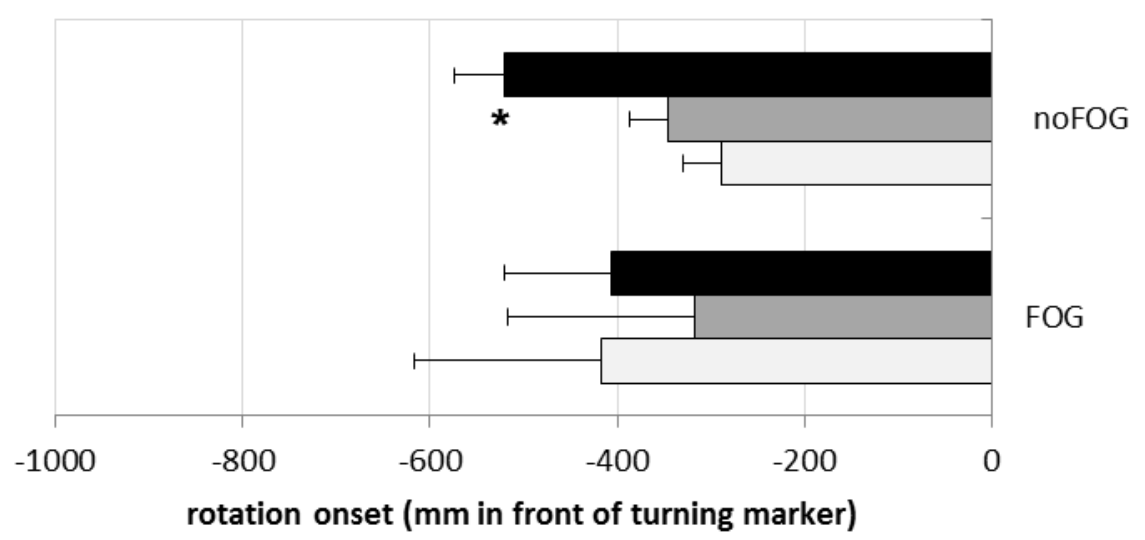

Figure 4: The onset of head $\square$, thorax $\square$ and pelvic $\square$ rotation expressed in mm distance to the retroreflective turning markers placed on the ground. (A) Differences between freezers, non-freezers and controls (ANOVA mixed model), (B) difference in controls between normal and slow speed (ANOVA repeated measures), (C) Difference in start of the turn between trials with and without FOG in the subgroup of 5 freezers who froze during the protocol (Friedman ANOVA). $*=p<0.05$ 
timing of maximum head-pelvis dissociation occurred at $89.7^{\circ}$ in trials without FOG, compared to at $106.4^{\circ}$ in FOG-trials. However, this tendency was not found to be significant $(\mathrm{p}=0.13)$.

\section{Influence of rigidity on kinematic variables}

No correlations (Spearman-Rank) were found between the neck-rigidity item of the UPDRS during OFF and turning parameters in non-freezers. In freezers, a higher neck-rigidity was correlated with an earlier initiation of pelvic rotation $(\mathrm{R}=0.62, \mathrm{p}=0.02)$ and tended to correlate with a smaller distance between the start of head and pelvic rotation $(\mathrm{R}=0.47, \mathrm{p}=0.11)$. This tendency towards a greater coupling between head and pelvis was also seen during the midturn, as the timing of maximum head-pelvis separation was delayed with increasing neckrigidity $(\mathrm{R}=0.62, \mathrm{p}=0.02)$.

\begin{tabular}{|l|l|l|l|l|}
\cline { 2 - 5 } \multicolumn{1}{c|}{} & \multicolumn{2}{c|}{ Freezers } & \multicolumn{2}{c|}{ Non-Freezers } \\
\cline { 2 - 6 } & Neck rigidity & p-value & Neck rigidity & p-value \\
\hline Start pelvic rotation & 0.62 & .02 & 0.02 & .96 \\
\hline Start head rotation & 0.09 & .78 & 0.16 & .59 \\
\hline Distance head-pelvis & 0.47 & .11 & 0.15 & .62 \\
\hline Timing MAX separation & 0.62 & .02 & 0.43 & .13 \\
\hline MAX separation & 0.19 & .53 & 0.22 & .44 \\
\hline
\end{tabular}

Table 3: Spearman-Rank correlation coefficients between neck rigidity and turning parameters in freezers and non-freezers.

\section{Discussion}

This study is the first to examine the differences in axial rotation between freezers, nonfreezers and controls and the possible link between "en-bloc"-turning and FOG. The turning- 
arc was equal in both groups and therefore, the axial movement differences between groups are the more striking.

\section{Turn preparation}

The start of the turn was characterized by a cranio-caudal sequence ${ }^{8,15,31}$, which was less pronounced in $\mathrm{PD}^{15}$. The current study shows that abnormal coordination during turning is even more pronounced in freezers, a finding which was not explained by disease-severity. We asked controls to mimic Parkinsonian gait speed and found that this cranio-caudal axial rotation pattern was then comparable between groups. This confirms previous findings that impaired axial rotation during walking is speed-dependent ${ }^{11,12,13}$, and raises the question whether turning en-bloc is merely a result of decreased gait speed and does not constitute a PD-specific phenomenon. As gait velocity differences between groups may have explained the delayed head rotation, it would be interesting to examine if asking subjects to turn more rapidly would lead to less "en-bloc"turning in all groups and less FOG in freezers. This is in line with earlier work showing that FOG reduced when patients turned faster using auditory cueing $^{32}$. When analyzing the FOG-trials separately, we found that the start of the turn was characterized by a complete "en-bloc" movement strategy, i.e. head and pelvis were moving almost simultaneously. This finding is particularly surprising given the fact that the FOGepisodes tended to happen much later in the turn, i.e. after the $90^{\circ}$ pivot point. No differences in step-length of the pre-turning steps were seen between trials with and without FOG in contrast to Chee et al. ${ }^{33}$, and only a tendency was seen for gait velocity. However, only 5 freezers froze during the protocol, resulting in limited power to describe differences between FOG and no FOG. The axial differences between trials with and without FOG were clearer, suggesting that en-bloc turning is more important in the etiology of FOG than step length.

The normal top-down coordination is considered to be an anticipatory navigation system to establish a stable frame of reference during turning ${ }^{31}$, aiding the visuomotor control necessary 
for the change of direction. Cowie et al. ${ }^{34}$ also suggested that a deficit of the online visuomotor system may contribute to the occurrence of FOG at doorways. The pedunculopontine nucleus (PPN) has been reported to play a significant role in head orientation in space ${ }^{35}$ and neural loss in PPN is related to $\mathrm{PD}^{36}$, and might be even more pronounced in freezers ${ }^{1,37}$. It is possible that the simultaneous start of head and pelvic rotation at the start of the turn might reflect impaired PPN activity when preparing for directional transition, eventually resulting in FOG.

\section{Axial movement impairment and FOG during turning}

The group differences of axial movement at onset also persisted during the continuation of the turn. Both the average (up till $65^{\circ}$ pelvic rotation) as well as the maximum head-pelvis separation was greater in controls compared to freezers and the timing of maximum separation was even earlier in controls and non-freezers. Most of those differences could be explained by differences in turn velocity, except for the timing of maximum separation which was group but not speed-dependent. Moreover, although not significantly, the timing of maximum separation tended to postpone in freezing-trials. Normally, after the point of maximum separation (at about $90^{\circ}$ ), head and pelvis start to rotate to each other (i.e. counterrotation) to prepare for regaining normal head-pelvis orientation in the new walking direction (see Figure 1). We found that this normal counter-rotation started later in the turn for freezers. We predicted that freezing-episodes would occur more frequently at the most pivotal point i.e. at about $90^{\circ}$ degrees when the head-pelvis separation is the greatest and turning possibly most unstable $^{21}$. Interestingly, FOG occurred mostly towards the end of the turn, when the difference in angular head-pelvis velocity was at its highest. This could be attributed to the delayed maximum head-pelvis separation as a result of which freezers were forced to complete their counter-rotation in a shorter section of the turn. When the required increase of normalized head-pelvis velocity difference to compensate for this delay was not reached, 
patients might have started to freeze because of insufficient preparation for the directional transition. This may explain why FOG occurs more frequently during 'continued' turning ${ }^{18,38}$, when patients are even less able to catch up with their spatial re-orientation. It is also possible that the observed axial movement changes during turning are merely part of the general lack of movement inherent to FOG or represent compensatory motor behavior, rather than being causative of freezing. The current pattern of the results on the localization of the freezing episodes within the turning arc needs to be confirmed in future studies in which more freezing episodes are obtained from patients with a wider range of freezing severity.

Despite the fact that FOG occurred at the end of the turn, FOG-duration lasted longer at the beginning of the turn. This suggests that patients may be able to re-initiate gait more easily from an almost symmetrical position rather than at the timing of maximal head-pelvis separation when gait is most asymmetrical. This is in line with Vaugoyeau et al., showing that gait initiation in combination with body re-orientation (i.e., stepping forward and diagonally) is more impaired than gait initiation alone (i.e., stepping forward) in freezers ${ }^{39}$.

\section{Rigidity}

Postural instability, axial rigidity and FOG are considered part of the PIGD phenotype of PD ${ }^{5}$. Macht et al. also showed that PD patients with rigidity as their main symptom had a higher chance to develop $\mathrm{FOG}^{3}$. On the contrary, Bartels et al. did not find a relationship ${ }^{40}$ between FOG and any of the cardinal symptoms of PD. The influence of rigidity on turning difficulties is at present unclear ${ }^{14,41}$. Mesure et al. suggested that the "en-bloc" movement is a compensatory mechanism for the impaired postural control during turning instead of a result of trunk rigidity ${ }^{10}$. In the current study, higher neck-rigidity was related to an increased 
coupling between head and pelvis during turning in freezers only. No association was apparent with the UPDRS balance-item but freezers had a higher fall frequency.

The present findings support the possibility that normalization of the impaired head-pelvis dissociation and the delayed counter-rotation might reduce FOG. This can theoretically be accomplished by encouraging freezers to first turn their head consciously when changing direction and initiating a turn. However, the efficacy of this strategy to reduce FOG and its impact on balance needs further study before clinical implementation can be recommended.

\section{Conclusions}

This study provides new insights into the role of axial movement in FOG. Turning in freezers was characterized by a closer coupling between head and pelvis and reduced head rotation to prepare for the directional transition, a pattern which became more pronounced during turns in which FOG occurred. These differences might be partially related to neck-rigidity. The changes of axial movement found during turning may have led to delayed counter-rotation of head and pelvis and contributed to the fact that FOG took place more frequently at the end of the turn.

\section{Acknowledgments}

Financial support was provided through the Flanders Fund for Scientific Research (project G0691.08) and a grant from the Research Council of Katholieke Universiteit Leuven, Belgium (contract OT/07/074). ). WV is Senior Clinical Investigator of Research Foundation - Flanders (FWO).

\section{Author Roles}

1. Research project: A. Conception, B. Organization, C. Execution; 
2. Statistical Analysis: A. Design, B. Execution, C. Review and Critique;

3. Manuscript Preparation: A. Writing of the first draft, B. Review and Critique;

Spildooren Joke: 1A, 1B, 1C, 2A, 2B, 3A

Vercruysse Sarah: 1A, 1B, 2C, 3B

Heremans Elke: 1B, 2C, 3B

Galna Brook: 1B, 2C, 3B

Vandenbossche Jochen: 1B, 2C, 3B

Desloovere Kaat: 1A, 1B, 2C, 3B

Vandenberghe Wim: 1A, 3B and patient recruitment

Nieuwboer Alice: 1A, 1B, 2A, 2C, 3B

\section{Financial disclosures for the past year}

Spildooren Joke

Stock ownership in medically related fields - none

Intellectual property rights - none

Consultancies - none

Expert testimony - none

Advisory boards -none

Employment: Research assistant KULeuven

Partnerships - none

Contracts - none

Honoraria - none

Royalties - none

Grants: Flanders Fund for Scientific Research (project G0691.08) and the Research Council of Katholieke Universiteit Leuven, Belgium (contract OT/07/074) 
Vercruysse Sarah

Stock ownership in medically related fields - none

Intellectual property rights - none

Consultancies - none

Expert testimony - none

Advisory boards -none

Employment - Research assistant KULeuven

Partnerships - none

Contracts - none

Honoraria - none

Royalties - none

Grants: Research Council of Katholieke Universiteit Leuven, Belgium (contract OT /07 / 074)

\section{Heremans Elke}

Stock ownership in medically related fields - none

Intellectual property rights - none

Consultancies - none

Expert testimony - none

Advisory boards -none

Employment - Postdoctoral researcher KULeuven

Partnerships - none

Contracts - none

Honoraria - none

Royalties - none 
Grants: PDM grant of the KULeuven

\section{Galna Brook}

Stock ownership in medically related fields - none

Intellectual property rights - none

Consultancies - none

Expert testimony - none

Advisory boards -none

Employment - Research associate Newcastle University

Partnerships - none

Contracts - none

Honoraria - none

Royalties - none

Grants - none

Vandenbossche Jochen

Stock ownership in medically related fields - none

Intellectual property rights - none

Consultancies - none

Expert testimony - none

Advisory boards -none

Employment - Research assistant VUBrussel

Partnerships - none

Contracts - none

Honoraria - none 
Royalties - none

Grants: Flanders Fund for Scientific Research (project G0691.08)

Desloovere Kaat

Stock ownership in medically related fields -

Intellectual property rights -

Consultancies -

Expert testimony -

Advisory boards -

Employment - Professor KULeuven

Partnerships -

Contracts -

Honoraria -

Royalties -

Grants: Flanders Fund for Scientific Research (project G0691.08) and the Research Council of Katholieke Universiteit Leuven, Belgium (contract OT /07/074)

Vandenberghe Wim

Stock ownership in medically related fields -

Intellectual property rights -

Consultancies -

Expert testimony -

Advisory boards -

Employment - Neurologist at the Movement Disorders Clinic of the University Hospital Leuven 
Partnerships -

Contracts -

Honoraria -

Royalties -

Grants: Senior Clinical Investigator of the Flanders Fund for Scientific Research

Nieuwboer Alice

Stock ownership in medically related fields - none

Intellectual property rights - none

Consultancies - none

Expert testimony - none

Advisory boards - none

Employment - Professor KULeuven

Partnerships -none

Contracts - none

Honoraria -none

Royalties -none

Grants: Flanders Fund for Scientific Research (project G0691.08) and the Research Council of Katholieke Universiteit Leuven, Belgium (contract OT /07 /074)

\section{References}

1. Nutt JG, Bloem BR, Giladi N, Hallett M, Horak FB, Nieuwboer A. Freezing of gait: moving forward on a mysterious clinical phenomenon. Lancet Neurol 2011; 10: 734-744.

2. Giladi N, McMahon D, Pzredborski S, et al. Motor blocks in Parkinson's Disease. Neurology 1992; 42: 333-339. 
3. Macht M, Kaussner Y, Möller JC, et al. Predictors of freezing in Parkinson's disease: A survey of 6620 patients. Mov Disord 2007; 22(7): 953-956.

4. Giladi N, Mc Dermott MP, Przedborski S, et al. Freezing of gait in PD: Prospective assessment in the DATATOP cohort. Neurology 2001; 56: 1712-1721.

5. Factor SA, Steenland NK, Higgins DS, et al. Postural instability/gait disturbance in Parkinson's disease has distinct subtypes: an exploratory analysis. J Neurol Neurosurg Psychiatry 2011; 82: 564-568.

6. Schaafsma JD, Balash Y, Gurevich T, Bartels AL, Hausdorff JM, Giladi N. Characterization of freezing of gait subtypes and the response of each to levodopa in Parkinson's disease. Eur Neurol 2003; 10: 391-398.

7. Stack E, Ashburn A, Jupp K. Strategies used by people with Parkinson's disease who report difficulty turning. Parkinsonism Relat Disord 2006; 12 :87-92.

8. Fuller JR, Adkin AL, Vallis LA. Strategies used by older adults to change travel direction. Gait Posture 2007; 25: 393-400.

9. Murray MP, Sepic SB, Gardner GM, Downs WJ. Walking patterns of men with Parkinsonism. Am J Phys Med 1978; 57(6): 278-294.

10. Mesure S, Azulay JP, Pouget J, Amblard B. Strategies of segmental stabilization during gait in Parkinson's disease. Exp Brain Res 1999; 129: 573-581.

11. Van Emmerik REA, Wagenaar RC, Winogrodzka A, Wolters EC. Identification of axial rigidity during locomotion in Parkinson's disease. Arch Phys Med Rehabil 1999; 80: 186191.

12. Van Emmerik RE, Wagenaar RC. Effects of walking velocity on relative phase dynamics in the trunk in human walking. J Biomech 1996; 29(9): 1175-1184. 
13. Lewek MD, Poole R, Johnson J, Halawa O, Huang X. Arm swing magnitude and asymmetry during gait in the early stages of Parkinson's disease. Gait Posture 2010; 31(2): 256-260.

14. Franzén E, Paquette C, Gurfinkel VS, Cordo PJ, Nutt JG, Horak FB. Reduced performance in balance, walking and turning tasks is associated with increased neck tone in Parkinson's disease. Exp Neurol 2009; 219(2): 430-438.

15. Crenna P, Carpinella I, Rabuffetti M, et al. The association between impaired turning and normal straight walking in Parkinson's disease. Gait Posture 2007; 26: 172-178.

16. Huxham F, Baker R, Morris ME, Iansek R. Head and trunk rotation during walking turns in Parkinson's disease. Mov Disord 2008; 23(10): 1391-1397.

17. Huxham F, Baker R, Morris ME, Iansek R. Footstep adjustments used to turn during walking in Parkinson's disease. Mov Disord 2008; 23(6): 817-823.

18. Spildooren J, Vercruysse S, Desloovere K, Vandenberghe W, Kerckhofs E, Nieuwboer A. Freezing of gait in Parkinson's Disease: the impact of dual-tasking and turning. Mov Disord 2010; 25(15): 2563-2570.

19. Hagen YJ, Snijders AH, Munneke M, Duysens J, Bloem BR. Rapid axial turns: the best way to provoke freezing of gait in parkinson's disease. Parkinsonism Relat Disord 2008; 14(suppl1): S26.

20. Salarian A, Zampieri C, Horak FB, Carlson-Kuhta P, Nutt JG, Aminian K. Analyzing 180 degrees turns using an inertial system reveals early signs of progression of Parkinson's disease. Conf Proc IEEE Eng Med Biol Soc. 2009; 2009: 224-227.

21. Huxham F, Gong J, Baker R, Morris M, Iansek R. Defining spatial parameters for nonlinear walking. Gait Posture 2006; 23: 159-163.

22. Hoehn MM, Yahr MD. Parkinsonism: onset, progression, and mortality. Neurology 1967; 17: 427-442. 
23. Folstein MF, Folstein SE, McHugh PR. Mini-mental state. A practical method for grading the cognitive state of patients for the clinician. J Psychiatr Res 1975; 12: 189-198.

24. Nieuwboer A, Rochester L, Herman T, et al. Reliability of the new freezing of gait questionnaire: agreement between patients with Parkinson's disease and their carers. Gait Posture 2009; 30: 459-463.

25. Movement Disorder Society Task Force on Rating Scales for Parkinson's Disease. The Unified Parkinson's Disease Rating Scale (UPDRS): status and recommendations. Mov Disord 2003; 18: 738-750.

26. Bowen A, Wenman R, Mickelborough J, Foster J, Hill E, Tallis R. Dual-task effects of talking while walking on velocity and balance following a stroke. Age Ageing 2001; 30: 319-323.

27. Canning CG, Ada L, Johnson JJ, McWhirter S. Walking capacity in mild to moderate Parkinson's Disease. Arch Phys Med Rehabil 2006; 87: 371-375.

28. Osaki Y, Kunin M, Cohen B, Raphan T. relative contribution of walking velocity and stepping frequency to the neural control of locomotion. Exp Brain Res 2008; 185: 121135.

29. Spildooren J, Vercruysse S, Meyns P, et al. Turning and unilateral cueing in Parkinson's disease patients with and without freezing of gait. Neuroscience 2012; 207: 298-306.

30. Chockalingam N, Chatterley F, Healy AC, Greenhalgh A, Branthwaite HR. Comparison of pelvic complex kinematics during treadmill and overground walking. Arch Phys Med Rehabil 2012 [Epub ahead of print]

31. Grasso R, prévost P, Ivanenko YP, Berthoz A. Eye-head coordination for steering of locomotion in humans: anticipatory synergy. Neuroscience Letters 1998; 253: 115-118.

32. Arias P, Cudeiro J. Effect of rhythmic auditory stimulation on gait in parkinsonian patients with and without freezing of gait. PLoS ONE 2010; 5: e9675. 
33. Chee R, Murphy A, Danoudis M, Georgiou-Karistianis N, Iansek R. Gait freezing in Parkinson's Disease and the stride length sequence effect interaction. Brain 2009; 132: 2151-2160.

34. Cowie D, Limousin P, Peters A, Hariz M, Day BL. Doorway-provoked freezing of gait in Parkinson's Disease. Mov Disord 2011 [Epub ahead of print]

35. Drew T, prentice S, Schepens B. Cortical and brainstem control of locomotion. Prog Brain Res 2004; 143: 251-261.

36. Lee MS, Rinne JO, Marsden CD. The pedunculopontine nucleus: Its role in the genesis of movement disorders. Yonsei Medical Journal 2000; 41(2): 167-184.

37. Snijders AH, Leunissen I, Bakker M, et al. Gait-related cerebral alterations in patients with Parkinson's Disease with freezing of gait. Brain 2011; 134: 59-72.

38. Snijders AH, Haaxma CA, Hagen YJ, Munneke M, Bloem BR. Freezer or non-freezer: Clinical assessment of freezing of gait. Parkinsonism Relat Disord 2011 [Epub ahead of print]

39. Vaugoyeau M, Viallet F, Mesure S, Massion J. Coordination of axial rotation and step execution: deficits in Parkinson's disease. Gait Posture 2003; 18: 150-157.

40. Bartels AL, Balash Y, Gurevich T, Schaafsma JD, Hausdorff JM, Giladi N. Relationship between freezing of gait (FOG) and other features of Parkinson's: FOG is not correlated with bradykinesia. J Clin Neuroscience 2003: 10(5): 584-588.

41. Schenkman M, Morey M, Kuchibhatla M. Spinal flexibility and balance control among community-dwelling adults with and without Parkinson's disease. J gerontol 2000; 55A(8): M441-445. 
